\title{
THE MEANING OF MASLOV'S ASYMPTOTIC METHOD: THE NEED OF PLANCK'S CONSTANT IN MATHEMATICS
}

\author{
BY JEAN LERAY
}

\begin{abstract}
H. Poincare defined asymptotic expansions. Their use by the $\mathbf{W}$. K. B. method introduced a new kind of solution of linear differential equations. Maslov showed their singularities to be merely apparent. The clarification of those results leads to the introduction of "Lagrangian functions", of their scalar product and of "Lagrangian operators", which constitutes a new structure: the "Lagrangian analysis". The last step of its definition requires the choice of a constant. That constant has to be Planck's constant, when the equation is the Schrödinger or the Dirac equation describing the hydrogen atom-the study of atoms with several electrons is very incomplete.
\end{abstract}

1. Henri Poincaré's main field, more precisely the one where the number of his publications is the highest, happens to be celestial mechanics. For instance, he tried to establish the convergence of the series by means of which the motion of the solar system is computed; it was a failure. He proved indeed the opposite: the divergence of those series, whose numerical values furnished the most impressive, precise and famous predictions in science during the last century! Henri Poincaré explained that paradox: those series give a very good approximation of the wanted result, provided only their first terms, namely, a reasonable number of them, are taken into account. Of course, demanding mathematicians to be reasonable is dubious but Henri Poincaré [4] made it clear by defining the asymptotic expansion $\sum_{n=0}^{\infty} a_{n} x^{n}$ of a function of $x$ at the origin: it is a formal series such that for each natural number $N$ there exists a positive number $c_{N}$ such that

$$
\left|f(x)-\sum_{n=0}^{N} a_{n} x^{n}\right| \leqslant c_{N}|x|^{N+1} \text { for } x \text { near } 0 .
$$

Thus an asymptotic expansion of $f$ is a formal series able to give a very good approximation of $f(x)$, when $x$ is small, but unable to supply the exact value of $f(x)$.

2. The W.K.B. method constructs asymptotic solutions of a linear differential equation

$$
H\left(x, \frac{1}{\nu} \frac{\partial}{\partial x}\right) u(\nu, x)=0 \quad\left(x \in X=\mathbf{R}^{l} ; \nu \in i[0, \infty[),\right.
$$

whose unknown is the function $u$ and whose parameter $\nu$ tends to $i \infty$.

Presented at the Symposium on the Mathematical Heritage of Henry Poincare in April, 1980; received by the editors June $15,1980$.

1980 Mathematics Subject Classification. Primary 47B99, 81C99; Secondary 35S99, $42 B 99$.

Key words and phrases. Fourier transform, Metaplectic group, Asymptotic solution, Lagrangian, Operator, Schrödinger, Dirac equation. 
Assume that (2.1) describes the evolution of a mechanical system; if that system were a finite set of particles, i.e., if (2.1) were an ordinary differential system, then Cauchy's problem should be studied. But let us assume it is a continuous mechanical system; therefore the physicists cannot impose its initial position and velocity: they have no more interest in Cauchy's problem.

What they are interested in are the waves

$$
U(\nu, x)=\alpha(\nu, x) e^{\nu \phi(x)},
$$

solutions of the equation (2.1); in (2.2), the phase $\phi$ is a real-valued function, $\nu$ is near $i \infty$; therefore $e^{\nu \phi}$ rapidly oscillates, whereas the amplitude $\alpha$ is a complex-valued function which slowly varies.

Therefore $\phi$ has to satisfy the first order nonlinear differential equation

$$
H\left(x, \phi_{x}\right)=0 \quad\left(\phi_{x}=\partial \phi / \partial x\right) .
$$

Assume $H$ to be a real-valued function of

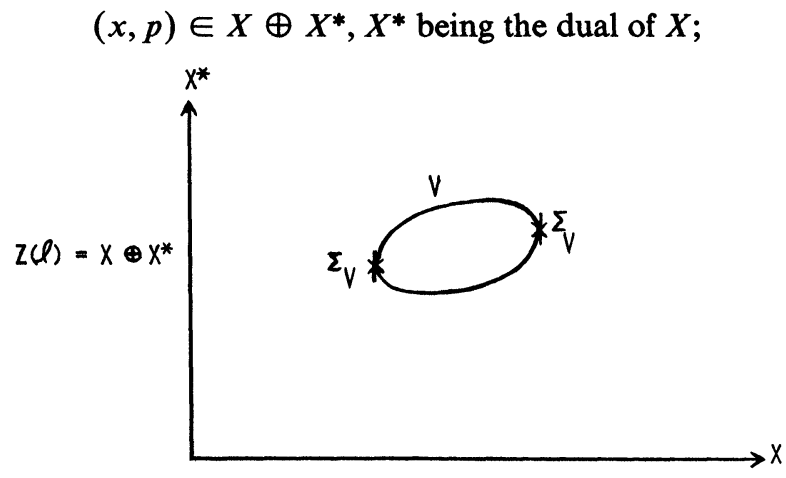

FIGURE 1

Denote by $W$ the hypersurface of $X \oplus X^{*}$ where $H$ vanishes :

$$
W: H(x, p)=0 \text {; assume }\left(H_{x}, H_{p}\right) \neq 0 \text { on } W \text {; }
$$

denote the graph of $\phi_{x}$ by

$$
V=\left\{\left(x, \phi_{x}\right)\right\} \subset X \oplus X^{*} .
$$

Then $V$ is any $l$-dimensional subvariety of $W$ on whose universal covering space $\check{V}$ there exists a function $\phi$ such that

$$
d \phi=\langle p, d x\rangle \quad\left(\langle p, x\rangle: \text { value of } p \in X^{*} \text { at } x \in X\right) ;
$$

i.e., $V$ is any $l$-dimensional subvariety of $W$ on which

$$
\sum_{j=1}^{l} d p_{j} \wedge d x_{j}=0
$$

$\left[\left(x_{j}\right),\left(p_{j}\right):\right.$ dual coordinates of $x \in X$ and $\left.p \in X^{*}\right]$.

Let $Z(l)$ be the $2 l$-dimensional vector space $X \oplus X^{*}$ provided with the symplectic structure $[\cdot, \cdot]$ defined as follows

$$
\text { if } z=(x, p) \text { and } z^{\prime}=\left(x^{\prime}, p^{\prime}\right) \text {, then }\left[z, z^{\prime}\right]=\left\langle p, x^{\prime}\right\rangle-\left\langle p^{\prime}, x\right\rangle \text {. }
$$


By definition, the Lagrangian subspaces of $Z(l)$ are its $l$-dimensional subspaces on which $[\cdot, \cdot]$ identically vanishes; the Lagrangian varieties of $Z(l)$ are its $l$-dimensional varieties whose tangent planes are Lagrangian. The definition (2.7) of $V$ means

$V$ is any Lagrangian variety contained in the hypersurface $W$.

The solution of the first order nonlinear differential equation (2.3) rests on the following fundamental property of $V$ : the variety $V$ is generated by characteristic curves of $W$, i.e., by curves of $W$ satisfying the Hamiltonian system

$$
\frac{d x_{j}}{H_{p_{j}}(x, p)}=-\frac{d p_{k}}{H_{x_{k}}(x, p)}
$$

$[j, k \in\{1, \ldots, l\} ; H$ is a first integral of that system].

Without discussing more completely the construction of $V$, assume $V$ and hence $\phi$ chosen: then (2.2) satisfies (2.1) $\bmod 1 / \nu$.

Now $U(\nu, x)$ satisfies

$$
H\left(x, \frac{1}{\nu} \frac{\partial}{\partial x}\right) U(\nu, x)=0 \bmod 1 / \nu^{N}
$$

[for any natural number $N$ ]

if and only if $\alpha(\nu, x)$ is a formal series in $1 / \nu$

$$
\alpha(\nu, x)=\sum_{r=0}^{\infty} \frac{1}{\nu^{r}} \alpha_{r}(x),
$$

the derivatives of $\alpha_{0}, \ldots, \alpha_{r}, \ldots$ along the characteristics generating $V$ having a known value, . . , a value depending on $\left(\alpha_{0}, \ldots, \alpha_{r-1}\right), \ldots$ Then $U(\nu, x)$, defined by (2.2), is a formal (with respect to $1 / \nu$ ) function of $x$ satisfying (2.11): it is said to be an asymptotic solution of (2.1); its above construction is called: W.K.B. method.

By that method wave mechanics is related to particle mechanics: indeed, the Hamiltonian system (2.10) could describe the motion of particles and the computation of $\alpha_{0}$ defines a density of those particles such that the conservation of their mass holds. Now the physicists hesitated between a corpuscular (Descartes and Newton, 17th century) and a wave (Huygens and Fresnel, 17th-19th centuries) structure of the light, before they concluded that light (Einstein, 1905: photons and quanta) and matter (de Broglie, 1924) have both structures. Henri Poincaré died ${ }^{1}$ in 1912 some years before it became clear that the quanta require a new physics, which has not yet been attained even today. So let us continue to study the asymptotic solutions of differential equations and thus apply to problems which arose before Henri Poincaré's death some of the concepts owing to him: algebraic topology, first homotopy group, covering spaces and groups.

(') He was only 58 years old; it was 3 years before "General Relativity" finally made "Relativity" acceptable by the astronomers. 
An asymptotic solution $U$ happens to be defined not on $X$ but on $V$, where it has singularities on the apparent contour $\Sigma_{V}$ of $V$, which is the set of the points of $V$ where $d x_{1} \wedge \cdots \wedge d x_{l}=0$, i.e., where $x$ cannot be used as local coordinate on $V$; the $\alpha_{r}$ become infinite on $\Sigma_{V}$; the order of their singularity increases with $r$.

For instance, let us use geometrical optics, i.e., use asymptotic solutions of the wave equation of light, for describing the propagation of the light issued from a monochromatic source through a steady optical system: the projections on $X$ of the characteristic curves generating $V$ are the rays of light, whose envelope, the "caustic", is the projection of $\Sigma_{V}$ on $X$; that caustic plays the role of image of the source; on it the asymptotic solution is no more defined; therefore geometrical optics should have no meaning beyond the caustic; however, it still holds. That paradox has been solved by V.P. Maslov [3] as follows.

The singularities of an asymptotic solution $U$ on the apparent contour $\Sigma_{V}$ are merely apparent singularities: indeed they are suppressed by a convenient Fourier transform; Maslov's proof needs to be clarified by V. I. Arnold's topological results [1] and by V.C. Buslaev's use [2] of I. E. Segal's metaplectic group [5], which contains the Fourier transforms used by Maslov. Those improvements finally do not give some deeper knowledge of the asymptotic behaviour of the functions; but they lead to a new structure, defining, for instance, quite a new type of solutions of differential equations; $\$ 3$ describes that structure, called Lagrangian analysis; \$4 discusses its applications.

\section{Lagrangian analysis.}

The symplectic geometry. Denote by $Z$ the symplectic space of dimension $2 l$ : i.e., $\mathbf{R}^{2 l}$ provided by a bilinear antisymmetric real-valued form $[\cdot, \cdot]$ of maximal rank. A frame $e^{2}$ is any isomorphism

$$
R: Z \rightarrow Z(l)=X \oplus X^{*}
$$

consistent with the symplectic structures of $Z$ and $Z(l)$ [see \$2].

If $R^{\prime}$ is another frame, then the change of frame $R R^{\prime-1}$ is any automorphism of $Z(l)$, that is, any element $s$ of the symplectic group $\operatorname{Sp}(l)$ :

$$
R R^{\prime-1} \in \operatorname{Sp}(l) \text {. }
$$

Each real-valued quadratic form $A$ of $\left(x, x^{\prime}\right) \in X \oplus X^{\prime}$, such that $\operatorname{det}\left(A_{x_{j} x_{k}^{\prime}}\right) \neq 0$, defines an element $s_{A}$ of $\operatorname{Sp}(l)$ as follows:

$$
\begin{aligned}
(x, p) & =s_{A}\left(x^{\prime}, p^{\prime}\right) \text { if and only if } \\
p & =A_{x}\left(x, x^{\prime}\right), \quad p^{\prime}=-A_{x^{\prime}}\left(x, x^{\prime}\right) ;
\end{aligned}
$$

\footnotetext{
${ }^{2} R$ is the initial of the French translation Repere of Frame. Let us recall that an orthonormed frame of the Euclidean space $E^{3}$ is nothing else but an isomorphism $E^{3} \rightarrow \mathbf{R} \oplus \mathbf{R} \oplus \mathbf{R}$, that direct sum being provided by the Euclidean structure implied by the structure of $\mathbf{R}$. That isomorphism has to be consistent with the Euclidean structures.
} 


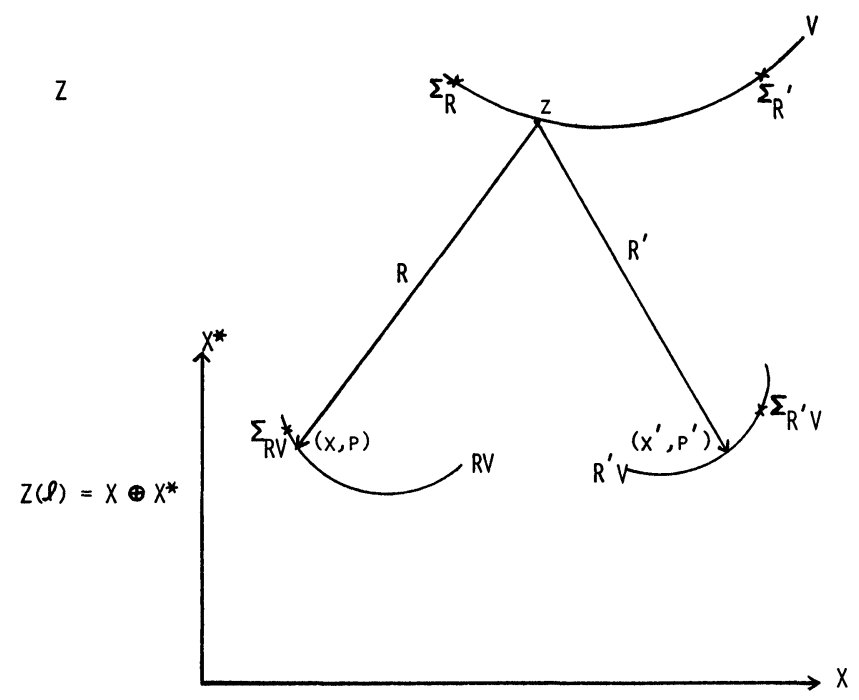

FIGURE 2

whence by Euler's formula

$$
A\left(x, x^{\prime}\right)=\langle p, x\rangle-\left\langle p^{\prime}, x^{\prime}\right\rangle
$$

$s_{A}$ is the generic element $s$ of $\operatorname{Sp}(l)$-but not any $s \in \operatorname{Sp}(l)$.

Lagrangian varieties $V$ are defined in $Z$ as they were in $Z(l)$ [see \&2]; that definition can be expressed as follows:

$$
d[z, d z]=0 \text { on } V
$$

i.e., on the universal covering space $\check{V}$ of $V$ there exists a real-valued function $\psi$, the Lagrangian phase, such that

$$
d \psi=\frac{1}{2}[z, d z]
$$

The image $R V$ of $V$ by $R$ is a Lagrangian variety of $Z(l)$; denote its phase [\$2] by $\phi_{R} \circ R^{-1}$; then, by a convenient choice of constants of integration

$$
\phi_{R}(\check{z})=\psi(\check{z})+\frac{1}{2}\langle p, x\rangle,
$$

where $z \in V$ is the projection of $\check{z} \in \check{V}$ and $(x, p)=R z$.

Hence, from (3.2), if $R R^{\prime-1}=s_{A}$ :

$$
\phi_{R}(\check{z})=\phi_{R^{\prime}}(\check{z})+A\left(x, x^{\prime}\right),
$$

where

$$
\begin{gathered}
(x, p)=R z, \quad\left(x^{\prime}, p^{\prime}\right)=R^{\prime} z, \\
z \in V \text { being the projection of } \check{z} \in \check{V} .
\end{gathered}
$$

The apparent contour $\check{\Sigma}_{R}$ of $\check{V}$ relative to $R$ is the subset of $\check{V}$ projecting on $\Sigma_{R}=R^{-1} \Sigma_{R V}$, which is the apparent contour of $V$, relative to $R$. In a sufficiently small neighborhood of each point of $\check{V} \backslash \check{\Sigma}_{R} \cup \check{\Sigma}_{R}$, the maps $\check{z} \mapsto z, z \mapsto x, z \mapsto x^{\prime}$ are diffeomorphisms. Now, since $R V$ is the graph of the 
gradient of its phase [see \$2]

$$
p=\partial \phi_{R} / \partial x, \quad p^{\prime}=\partial \phi_{R^{\prime}} / \partial x^{\prime}
$$

thus, by $(3.1)_{2}$, the diffeomorphism $x \mapsto x^{\prime}$ can be defined by

$$
\left[A\left(x, x^{\prime}\right)+\phi_{R^{\prime}}\right]_{x^{\prime}}=0 \text {, assuming (3.6). }
$$

The group $\operatorname{Sp}_{2}(l)$. Define a formal function on $\check{V} \backslash \check{\Sigma}_{R^{\prime}}$ by the choice of a frame $R^{\prime}$ and by an expression

$$
U_{R^{\prime}}(\nu, \check{z})=\alpha_{R^{\prime}}(\nu, \check{z}) e^{\nu \phi_{R^{\prime}}(\check{z})},
$$

where $\alpha_{R^{\prime}}$ is a formal series

$$
\alpha_{R^{\prime}}(\nu, \check{z})=\sum_{r=0}^{\infty} \frac{1}{\nu^{r}} \alpha_{r}^{\prime}(\check{z}),
$$

the $\alpha_{r}^{\prime}$ being infinitely derivable functions: $\check{V} \backslash \check{\Sigma}_{R^{\prime}} \rightarrow \mathbf{C}$.

From now on, let $A$ be the datum of

(i) a real-valued quadratic form $A$ of $\left(x, x^{\prime}\right) \in X \oplus X$;

(ii) a choice of $\Delta(A)= \pm\left[\operatorname{det}\left(A_{x_{j} x_{k}^{\prime}}\right)\right]^{1 / 2}$; we assume: $\Delta(A) \neq 0$. by

Let $R=s_{A} R^{\prime}$; i.e., $R R^{\prime-1}=s_{A} \in \mathrm{Sp}(l)$; define a linear map $S_{A}: U_{R^{\prime}} \mapsto U_{R}$

$$
\begin{aligned}
\left(S_{A} U_{R^{\prime}}\right)(\nu, \check{z}) & =U_{R}(\nu, \check{z}) \\
& =\left(\frac{|\nu|}{2 \pi i}\right)^{1 / 2} \Delta(A) \int_{\check{V}_{\check{L}} \check{\Sigma}_{R^{\prime}}} e^{\nu A\left(x, x^{\prime}\right)} U_{R^{\prime}}\left(\nu, \check{z}^{\prime}\right) d^{l} x^{\prime},
\end{aligned}
$$

where

$$
\begin{aligned}
& z \in V \text { is the projection of } \check{z} \in \check{V} ;(x, p)=R z ; \\
& z^{\prime} \in V \text { is the projection of } \check{z}^{\prime} \in \check{V} ;\left(x^{\prime}, p^{\prime}\right)=R^{\prime} z^{\prime} .
\end{aligned}
$$

Obviously that map $S_{A}$ can be decomposed into:

(i) the product by $e^{\nu A(0, \cdot)}$;

(ii) a map of type (3.9), where $A$ is a bilinear, real-valued function of $\left(x, x^{\prime}\right) \in X \oplus X$, so that it is essentially a Fourier transform, which is unitary thanks to the numerical factor in (3.9);

(iii) the product by $e^{\nu A(\cdot, 0)}$.

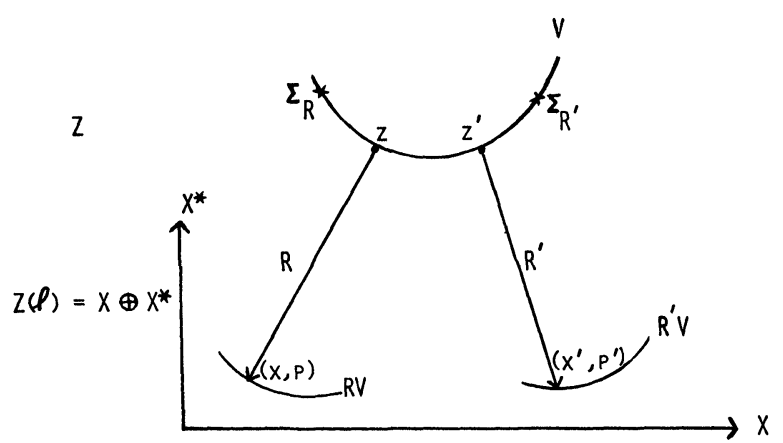

Figure 3 
According to Maslov, the integral in (3.9) has to be computed by the method of the stationary phase (neglecting the terms more rapidly decreasing than any power of $1 / \nu$, when $\nu$ tends to $i \infty)$. The phase of the integrand is $A\left(x, x^{\prime}\right)+\phi_{R^{\prime}}\left(\check{z}^{\prime}\right)$; assuming that $\operatorname{Supp}\left(U_{R^{\prime}}\right)$ belongs to $\check{V} \backslash \check{\Sigma}_{R} \cup \check{\Sigma}_{R^{\prime}}$ and is small, the assumption that this phase is stationary means that (3.7) holds; then $z=z^{\prime}$; hence, from (3.5): $U_{R}$ is a formal function on $\check{V}$, related to $R$; the linear transform $S_{A}$ is local on $\check{V}: \operatorname{Supp}\left(U_{R}\right)=\operatorname{Supp}\left(U_{R^{\prime}}\right)$.

It can be shown that the map $S_{A}$ of formal functions is the generic-but not any-element of the covering group of order $2, \mathrm{Sp}_{2}(l)$, of $\mathrm{Sp}(l)$; the projection $\mathrm{Sp}_{2}(l) \rightarrow \mathrm{Sp}(l)$ maps the two $S_{A}$ corresponding to opposite choices of $\Delta(A)$ onto the same $s_{A}$. Hence

LEMma. Any $S \in \mathrm{Sp}_{2}(l)$, with projection $s \in \mathrm{Sp}(l)$, maps each formal function $U_{R^{\prime}}$ defined on $V \backslash \check{\Sigma}_{R} \cup \dot{\Sigma}_{R^{\prime}}$ into such a formal function $U_{R}$, where $R=s R^{\prime}$. That mapping is local.

Comment. Of course the preceding account is not a proof of that lemma, which follows from the following results. Let $\mathcal{S}(X)$ be the Schwartz space of functions: $X \rightarrow \mathbf{C}$, all of whose derivatives decrease rapidly at infinity; its dual is the Schwartz space $\mathcal{S}^{\prime}(X)$ of tempered distributions; let $\mathcal{H}(X)$ be the Hilbert space of square-integrable functions: $X \rightarrow \mathbf{C}$;

$$
\delta(X) \subset \mathcal{H}(X) \subset \mathcal{S}^{\prime}(X)
$$

Each $S \in \operatorname{Sp}_{2}(l)$ defines a continuous automorphism of $\mathcal{S}^{\prime}(X)$; its restriction to $\mathcal{H}(X)$ is a unitary automorphism of $\mathcal{H}(X)$; its restriction to $\mathcal{S}(X)$ is a continuous automorphism of $\delta(X)$, which is defined by (3.9) if $S=S_{A}$. Then each $S$ defines also an automorphism of the vector space whose elements are the classes of functions of $(x, \nu)$ having the same asymptotic behaviour when $\nu$ tends to $i \infty$; any formal function $U$, defined on $X$ by

$$
\begin{aligned}
U(\nu, x) & =\alpha(\nu, x) e^{\phi \nu(x)}, \\
\text { where } \alpha(\nu, x) & =\sum_{r=0}^{\infty} \frac{1}{\nu^{r}} \alpha_{r}(x), \quad \alpha_{r}: X \rightarrow \mathbf{C}, \phi: X \rightarrow \mathbf{R},
\end{aligned}
$$

specifies one of those asymptotic classes; now $\mathrm{Sp}_{2}(l)$ does locally operate [see: lemma], if lifted from $X$ to $\check{V}$, by means of the use of formal functions $U_{R}$ on $\check{V}$ and of their projections $U$ defined by

$$
U(\nu, x)=\sum_{\check{z}} U_{R}(\nu, \check{z}), \quad \text { where } x \text { and } \check{z} \text { have to satisfy (3.6) }
$$

(that lifting assumes the support of $U_{R}$ to be small enough).

Comment. The two $S \in \mathrm{Sp}_{2}(l)$ having the same projection $s \in \mathrm{Sp}(l)$ may be denoted by $\pm S$. Denote by $c \in S^{1}$ the product of a function by the complex number $c$ of norm 1 . The elements of $I$. E. Segal's metaplectic group [5] are the $c S$, assuming $c S=c^{\prime} S^{\prime}$, if and only if $c^{\prime}= \pm c, S^{\prime}= \pm S( \pm$ being the same).

The $q$-symplectic geometry enables an improvement of the preceding lemma. Denote by $\Lambda$ the Lagrangian Grassmannian of $Z$, i.e., the set of its Lagrangian vector subspaces; $V$. I. Arnold [1] established the isomorphism of 
its Poincaré group (i.e., first homotopy group) with the additive group of the integers

$$
\pi_{1}[\Lambda] \simeq \mathbf{Z}
$$

Thus, for any $q \in\{1,2, \ldots, \infty\}, \Lambda$ has a unique covering space $\Lambda_{q}$ of order $q$. The choice of a $\lambda_{q} \in \Lambda_{q}$ with a given projection $\lambda$ on $\Lambda$ is said to be the choice of a $q$-orientation of that $\lambda$; the 2-orientation is the usual Euclidean orientation. Similarly define $\Lambda(l)$ and $\Lambda_{q}(l)$ for $Z(l)$.

Moreover,

$$
\pi_{1}[\operatorname{Sp}(l)] \simeq \mathbf{Z}
$$

thus, for any $q, \mathrm{Sp}(l)$ has a unique covering group $\mathrm{Sp}_{q}(l)$ of order $q$. Now $S \in \operatorname{Sp}_{q}(l)$ induces a homeomorphism of $\Lambda_{2 q}(l)$, which has a projection on $\Lambda(l)$, namely, the homeomorphism of $\Lambda(l)$ induced by the projection $s$ of $S$ onto $\operatorname{Sp}(l)$.

Let us define a $q$-frame $R$ as being

(i) an isomorphism $Z \rightarrow Z(l)$, consistent with the symplectic structures;

(ii) a homeomorphism $\Lambda_{2 q} \rightarrow \Lambda_{2 q}(l)$, having a projection which is the homeomorphism $\Lambda \rightarrow \Lambda(l)$ induced by the preceding isomorphism.

Then a change of $q$-frame $R R^{\prime-1}$ is

(i) an element $s$ of $\mathrm{Sp}(l)$;

(ii) a homeomorphism of $\Lambda_{2 q}(l)$ induced by one of the $S \in \operatorname{Sp}_{q}(l)$, whose projection is $s \in \mathrm{Sp}(l)$.

Hence, that change of $q$-frame $R R^{\prime-1}$ may be identified with that $S \in$ $\operatorname{Sp}_{q}(l):$

$$
R R^{\prime-1}=S \in \operatorname{Sp}_{q}(l) ; \text { i.e., } R=S R^{\prime} \text {. }
$$

Let us choose $q=2$ and make more precise the definition of a formal function $U_{R^{\prime}}$ on $\check{V}$ : from now on, $R^{\prime}$ has to be a 2-frame; then the definition of $U_{R}=S U_{R^{\prime}}$ has to be supplemented as follows:

$$
R=S R^{\prime} \text {. }
$$

Thus the statement of the preceding lemma is simplified as follows:

THEOREM. Any $S \in \mathrm{Sp}_{2}(l)$ maps any formal function $U_{R^{\prime}}$ defined on $\check{V} \backslash \check{\Sigma}_{R}$ $\cup \check{\Sigma}_{R^{\prime}}$ into such a formal function $U_{R}$, where $R=S R^{\prime}$. That mapping is local.

The preceding theorem makes the following definition possible.

Lagrangian functions on $\check{V}$. Let $V$ be a Lagrangian variety in $Z$. If for each 2-frame $R$ a formal function $U_{R}$ is given on $\check{V} \backslash \check{\Sigma}_{R}$ and if

$$
\left(\forall R, R^{\prime}\right) \quad U_{R}=S U_{R^{\prime}} \text { on } \check{V} \backslash \check{\Sigma}_{R} \cup \check{\Sigma}_{R^{\prime}} \text {, where } S=R R^{\prime-1} \text {, }
$$

then the set of those $U_{R}$ constitutes a Lagrangian function $\check{U}$ on $\check{V}$ :

$$
\check{U}=\left\{U_{R}\right\} \text {, by definition; }
$$

$U_{R}$ is said to be the expression of $\check{U}$ in the 2-frame $R$. Obviously $\check{U}$ has a support and a restriction to each open subset of $\check{V}$ :

$$
\left(\check{V} \backslash \check{\Sigma}_{R}\right) \cap \operatorname{Supp}(\check{U})=\operatorname{Supp}\left(U_{R}\right)
$$


COMment. $U_{R}$ has on $\check{\Sigma}_{R}$ singularities, which can be described, using [3] Maslov's index $m_{R}$ : it is a locally constant function $\check{V} \backslash \check{\Sigma}_{R} \rightarrow \mathbf{Z}$, whose topological definition (by means of Kronecker's index; i.e., intersection of chains) is due to V.I. Arnold [1] and which has also a geometric definition.

The scalar product $\left(\cdot{ }^{\cdot} \cdot\right)$ of two Lagrangian functions with compact supports can be defined, using the facts that $\operatorname{Sp}_{2}(l)$ is unitary on $\mathcal{H}(X)$ and that there are partitions of the unity into sums of Lagrangian operators with supports in elements of any given open covering of $Z$.

Lagrangian operators. Let $\Omega$ be an open subset of $Z$; define on $\Omega$ a formal function, without phase

$$
a^{0}(\nu, z)=\sum_{r=0}^{\infty} \frac{1}{\nu^{r}} a_{r}^{0}(z)
$$

$a_{r}^{0}$ being an infinitely derivable function $\Omega \rightarrow$ C. For each frame $R$, define

$$
a_{R}^{0}(\nu, x, p)=a^{0}(\nu, z), \quad \text { where }(x, p)=R z,
$$

and then

$$
a_{R}^{ \pm}(\nu, x, p)=\exp \left( \pm \frac{1}{2 \nu}\langle\partial / \partial x, \partial / \partial p\rangle\right) a_{R}^{0}(\nu, x, p) .
$$

First, assume $a^{0}$, i.e., the $a_{r}$, to be polynomial; define the formal differential operator with polynomial coefficients

$$
a_{R}=a_{R}^{+}\left[\nu, \stackrel{(2)}{x}, \frac{1}{\nu} \frac{(1)}{\partial x}\right]=a_{R}^{-}\left[\nu, \stackrel{(1)}{x}, \frac{1}{\nu} \frac{\stackrel{(2)}{\partial}}{\partial x}\right],
$$

(1) specifying the operators to be applied at first; $a_{R}$ locally maps a formal function $U$ defined on $X$ [see (3.10)] onto another one of same type and, by means of the projection (3.11) of $U_{R}$ onto $U$, a formal function $U_{R}$ defined on $\check{V} \backslash \check{\Sigma}_{R}$ onto another one. The set of the $a_{R}$ constitutes a Lagrangian operator: $a=\left\{a_{R}\right\}$, by definition; if $\check{U}=\left\{U_{R}\right\}$ is a Lagrangian function defined on $\check{V}$, then $\left\{a_{R} U_{R}\right\}$ is also such a function, denoted by

$$
a \check{U}=\left\{a_{R} U_{R}\right\} \text {. }
$$

Now if formal polynomials tend on $\Omega$ to a formal function $a^{0}$, then their associated Lagrangian operators have a limit: the Lagrangian operator $a$ associated to the formal function $a^{0}$ defined on $\Omega$.

If $\breve{U}$ is a Lagrangian function defined on $\check{V}$ and if $V \subset \Omega$, then a maps locally $\check{U}$ onto $a \check{U}$ defined on $\check{V}$.

Assume

$$
(\forall z \in \Omega) \quad a_{0}^{0}(z) \neq 0 ;
$$

then $a$ has an inverse $a^{-1}$, which is still a Lagrangian operator.

4. Lagrangian functions on $V$ : The need of Planck's constant. The definition of Lagrangian functions on $V$ makes use of Lagrangian functions on $\check{V}$ and of Poincaré's group $\pi_{1}[V]$ of $V$. 
Each $\gamma \in \pi_{1}[V]$ defines a homeomorphism of $\check{V}:$ if $\check{z} \in \check{V}$, then $\gamma \check{z} \in \check{V}, \check{z}$ and $\gamma \check{z}$ having the same projection on $V . \phi_{R} \circ \gamma-\phi_{R}$ is a constant, namely, the period $c_{\gamma}=\frac{1}{2} \int_{\gamma}[z, d z]$ of $\psi$. Therefore, if $U_{R}=\alpha_{R} e^{\nu \phi_{R}}$ is a formal function defined on $\check{V}$, then

$$
U_{R} \circ \gamma^{-1}=\alpha_{R} \circ \gamma^{-1} e^{\nu \phi_{R}-v c_{\gamma}}
$$

is not a formal function defined on $\check{V}$; indeed, its phase is not $\phi_{R}$, but $\phi_{R}-c_{\gamma}$. Obviously the simplest definition of the image, $\gamma U_{R}$, which has to be a formal function on $\check{V}$, of $U_{R}$ by $\gamma$ is

$$
\gamma U_{R}=e^{\left(\nu-\nu_{0}\right) c_{\gamma}} U_{R} \circ \gamma^{-1},
$$

$\nu_{0}$ being a constant we have to choose; since $\nu$ is a purely imaginary variable tending to $i \infty$, let us choose

$$
\nu_{0} \in i[0, \infty[
$$

and note that there is no reason for making some more specific choice, for instance $\nu_{0}=0$.

In the quantum mechanics, the choice agreeing with the experimental results will be [see §5]

$$
\nu_{0}=\frac{i}{\hbar}=\frac{2 \pi i}{h}, \quad h \text { being Planck's constant } .
$$

Once $\nu_{0}$ is chosen, (4.1) shows how Poincaré's group $\pi_{1}[V]$ maps formal functions $U_{R}$, defined on $\check{V}$ and related to $R$, onto formal functions of the same kind; $\pi_{1}[V]$ commutes with $\operatorname{Sp}_{2}(l)$; therefore $\pi_{1}[V]$ maps Lagrangian functions $\breve{U}$ on $\breve{V}$ onto Lagrangian functions on $\check{V}$

$$
\text { if } \check{U}=\left\{U_{R}\right\}, \text { then } \gamma \check{U}=\left\{\gamma U_{R}\right\} \text {. }
$$

The group $\pi_{1}[V]$ commutes with the Lagrangian operators: $a(\gamma \check{U})=\gamma(a \check{U})$.

Define the Lagrangian functions on $V$ as those Lagrangian functions $U=\left\{U_{R}\right\}$ on $\check{V}$ which are invariant by $\pi_{1}[V]$, i.e., such that for some $R$ and hence for any $R$ :

$U_{R}^{0}=\alpha_{R} e^{\nu_{0} \phi_{R}}$ is a function defined on $V$, with formal numerical values.

A Lagrangian operator $a$ transforms locally a Lagrangian function $U$ on $V$; $a U$ is a Lagrangian function on $V$. A Lagrangian function $U$ on $V$ has a support

$$
\operatorname{Supp}(U) \subset V
$$

Define

$$
\operatorname{Supp}(a)=\operatorname{Supp}\left(a^{0}\right) \subset Z,
$$

where the Lagrangian operator $a$ is associated with the formal function $a^{0}$. Then

$$
\operatorname{Supp}(a U) \subset \operatorname{Supp}(a) \cap \operatorname{Supp}(U) .
$$

The scalar product $\left(\cdot \Gamma^{\cdot}\right)$ of two Lagrangian functions on $V$ and $V^{\prime}$ can be defined, when the intersection of their supports is compact; that definition makes use of $(\cdot Y \cdot)$ which essentially differs from $(\cdot Y \cdot)$. 
$(\cdot Y \cdot)$ defines only a quasi-norm: if $\check{U}$ is defined on $\check{V}$, then $(\check{U} \mid \check{U})=0$ does not imply $\check{U}=0$.

$(\cdot \cdot \cdot)$ defines a norm $\|U\|$, which is a positive formal number without phase

$$
\|U\|=\sum_{r=0}^{\infty} \frac{1}{\nu^{r}} \alpha_{r}, \quad \text { where } \alpha_{r} \in \mathbf{C} ;
$$

"positive" means $i^{-r} \alpha_{r}$ real, $i^{-s} \alpha_{s}>0, s$ being the smallest $r$ such that $\alpha_{r} \neq 0$.

Schwarz's inequality and triangle inequality hold for those scalar products, quasi-norm and norm, strictly for $(\cdot \cdot \cdot)$.

Now the definition of the new structure called Lagrangian analysis is achieved.

Nonhomogeneous Lagrangian equation. Let

$$
a U=U^{\prime}
$$

be that equation, where $a$ is the Lagrangian operator associated with the given formal function

$$
a^{0}=\sum_{r=0}^{\infty} \frac{1}{\nu^{r}} a_{r}^{0} \quad\left(a_{r}^{0}: \Omega \rightarrow \mathbf{C}\right),
$$

where $U^{\prime}$ is a given Lagrangian function on $V \subset \Omega$, the unknown Lagrangian function $U$ having, obviously, to be defined on $V$. Assume

$$
(\forall z \in \Omega) \quad a_{0}^{0}(z) \neq 0 ;
$$

then $a$ has an inverse $a^{-1}$, which is a Lagrangian operator defined on $\Omega$; therefore (4.8) has the unique solution

$$
U=a^{-1} U^{\prime}
$$

The homogeneous Lagrangian equation

$$
a U=0
$$

is much less trivial: $U$ is defined on a Lagrangian variety $V$ belonging to the variety $W$ where $a_{0}^{0}$ vanishes; assume $a_{0}^{0}$ real-valued; denote $a_{0}^{0}=H$; then $W$ is the hypersurface of $Z$ defined by the equation

$$
W: H(z)=0 ;
$$

on each Lagrangian variety $V \subset W$, the solutions of (4.10) can be locally constructed by the W.K.B. method; the complete discussion of (4.10) is never easy, except for $l=1$.

The homogeneous Lagrangian system

$$
(\forall j=1,2, \ldots, l) \quad a^{(j)} U=0
$$

is especially interesting under the following assumptions: the $l$ Lagrangian operators $a^{(j)}$ commute and are associated with real-valued functions $H^{(j)}$ defined on a domain $\Omega$ of $Z$. Then $U$ has to be defined on the variety $V$ of $Z$ whose equations are

$$
V: H^{(1)}(z)=\cdots=H^{(l)}(z)=0 ;
$$


that variety is Lagrangian; indeed the $H^{(j)}$ are in involution (i.e., their Poisson brackets vanish); the amplitude $\alpha_{R}$ of each expression $U_{R}$ of $U$ is obtained by the integration on $V$ of closed Pfaffian forms.

\section{Application to the quantum mechanies of the hydrogen atom in a constant magnetic field.}

Schrödinger's equation. Let $H$ be the Hamiltonian of the electron belonging to that atom; i.e., the Hamiltonian system (2.10) describes the nonquantified trajectories of that nonrelativistic or relativistic electron; then the Lagrangian operator $a$ associated to $H$ is the Schrödinger operator or the relativistic Schrödinger operator, i.e., the Klein-Gordon operator. $H$ and, therefore, $a$ depend on a parameter $E$, which is the energy level of the electron.

The quantum mechanics asserts that $E$ has a value such that the equation

$$
a U=0
$$

has a nontrivial solution.

In wave quantum mechanics, the numerical value $\nu_{0}=i / \hbar$ is given to $\nu, U$ is a function $X \rightarrow \mathrm{C}, U$ and its gradient $U_{x}$ have to be square-integrable. $U$ represents a not-observable quantity.

That fact allows us to assume that $U$ is a Lagrangian function defined on a Lagrangian variety $V$. Replace the assumption of square-integrability by the following one: $V$ is compact. Note that the equation (5.1) has always to be supplemented by two other equations connected to the length of the momentum of the impulse and its component in the direction of the magnetic field. Therefore $U$ has to satisfy a system of three Lagrangian equations. That system belongs to the type (4.12).

That Lagrangian system and the classical system studied in wave quantum mechanics are not equivalent, but the constructions of their solutions have some likeness and finally both of them define the same energy levels $E$.

Their computation becomes extremely direct and simple if the Lagrangian system is used $\bmod 1 / \nu^{2}$ and therefore $U$ defined $\bmod 1 / \nu$.

Dirac's equation can be likewise solved in Lagrangian analysis; that gives anew the standard energy levels, even when the magnetic field is so strong that the Zeeman effect is replaced by the much more complicated PaschenBack effect.

6. The study of atoms with several electrons by the wave quantum mechanics requires the computing of eigenvalues, which cannot be carried out, except in a few cases, or by using crude approximations suggested by the spirit of the first quantum mechanics. Until now the Lagrangian analysis did not offer better possibilities; see Maslov and his collaborators about the use of crude approximations.

\section{BIBLIOGRAPHY}

1. V. I. Arnold, On a characteristic class intervening in quantum conditions, Functional Anal. Appl. 1 (1967), 1-14. (Russian with English transl.)

2. V. C. Buslaev, Quantization and the W. K. B. method, Trudy Mat. Inst. Steklov 110 (1970), 5-28. (Russian) 
3. V. P. Maslov, Perturbation theory and asymptotic methods, M. G. U., Moscow, 1965. (Russian)

4. H. Poincaré, Sur les intégrales irrégulières des équations linéaires, Acta Math. 8 (1886), 295-344.

5. I. E. Segal, Foundations of the theory of dynamical systems of infinitely many degrees of freedom. I, Mat.-Fys. Medd. Danske Vid. Selsk. 31 (1959), 1-39.

6. J. Leray, Analyse lagrangienne et mécanique quantique, Séminaire du Collège de France 1976-1977; R. C. P. 25, Strasbourg, 1978.

The present paper is a comment of the preceding preprint, a revised version of which will be published in English by M. I. T. Press, Cambridge, Massachusetts and in Russian by M. I. R., Moscow.

Collìge de France, (Math.) F-75231 Paris Cedex 05, France 
